Br J Dev Psychol. 2016 March ; 34(1): 1-5. doi:10.1111/bjdp.12138.

\title{
The Development of Action Mirroring
}

\author{
Kimberly Cuevas and \\ Department of Psychological Sciences, University of Connecticut, 99 East Main St., Waterbury, \\ CT 06702, USA \\ Markus Paulus \\ Developmental Psychology, Ludwig-Maximilians-Universität München, 80802 Munich, Germany \\ Kimberly Cuevas: kimberly.cuevas@uconn.edu
}

\begin{abstract}
The past decade has experienced an increasing interest in children's mirroring of others' behavior. Behavioral investigations have focused on the development and significance of mimicry and imitation. Others have focused on the neural basis of action mirroring, identifying particular electrophysiological markers or related brain regions (e.g., Cuevas, Cannon, Yoo, \& Fox, 2014). A vivid debate concerns the functional mechanisms that subserve and lead to action mirroring: some have argued for an impact of low-level actionperception couplings (e.g., Heyes, 2010; Paulus, 2014), others have suggested that action mirroring is the consequence of higher-level processes (e.g., Csibra, 2007), and again others have discussed a potential innate basis of mirroring (e.g., Lepage \& Theoret, 2007). Finally, the consequences of action mirroring for social functioning have been discussed with respect to its role in action understanding and fostering social relations (e.g., Over \& Carpenter,
\end{abstract} 2012).

One point of debate concerns the underlying mechanisms. This has largely focused on the ontogeny of mirroring (e.g., Jones, 2007; Meltzoff, 2007) and the neural basis of action mirroring with a particular focus on the so-called mirror neurons. The discovery of mirror neurons in rhesus macaques revealed one way in which action perception and execution were potentially linked (cf. Rizzolatti \& Craighero, 2004). Subsequent work with humans has indicated the existence of neural mirroring systems, with evidence of neural mirroring activity during infancy (see Cuevas et al., 2014, for review). Yet, much theoretical debate surrounds the origin of neural mirroring systems. From a genetic (i.e., phylogenetic, adaptation) perspective, initial variability in the predisposition for mirror neurons, resulted in some organisms having advantages in action understanding (Rizzolatti \& Arbib, 1998). The subsequent consequences of natural selection have resulted in a nearly universal genetic predisposition for mirror neurons. In other words, according to this account, infants are born with mirror neurons that are shaped throughout development via subsequent motor and sensory experiences (Bonini \& Ferrari, 2011). More recently, epigenetic (evo-devo) accounts have been proposed to account for variations in mirror neuron activity, including contributions of environmental differences early in development (Ferrari, Tramacere,

Correspondence concerning this article should be addressed to Kimberly Cuevas, Department of Psychological Sciences, University of Connecticut, 99 East Main St., Waterbury, CT 06702; Phone: 203-236-9823; kimberly.cuevas@uconn.edu. 
Simpson, \& Iriki, 2013). An associative learning account, on the other hand, argues that infants are not born with mirror neurons. Rather, infants have genetic predispositions for domain-general associative learning and connectivity between sensory and motor cortical regions. Mirror neurons emerge from motor neurons via correlated sensorimotor experience (i.e., co-activation of sensory and motor neurons) throughout the course of typical development (Heyes, 2014; Paulus, 2014). These proposals relate to suggestions that imitation itself is the result of learning processes (Jones, 2009).

A related question concerns what is actually mirrored. Is mirroring taking place even at finegrained kinematic levels or does mirroring happen at more abstract levels that specify merely the effector involved or even more abstract units? This question is not only an empirical issue, but also relates to conceptual questions of how to demarcate imitation from related phenomena such as mimicry or emulation (e.g., Paulus, 2011; Tomasello, 1999). Likewise, given that research with adults suggested that people do not only mirror actions, but also others' sensory inputs (mirror touch; e.g., Ebisch et al., 2008), a broader investigation of the breadth and limits of mirror phenomena in development seems to be warranted.

Another highly debated topic involves the role of action mirroring in action understanding and social functioning. According to the direct-matching hypothesis (Rizzolatti, Fogassi, \& Gallese, 2001), mirror neurons permit the observer to directly understand another's action (i.e., embodied simulation). Thus, action understanding occurs through a bottom-up process. In contrast, the teleological reasoning hypothesis (Csibra \& Gergely, 2007) considers action understanding to emerge from a top-down process (i.e., outside the motor system). The observer evaluates actions in terms of goals and subgoals and action mirroring occurs thorough an emulative processes (Csibra, 2007). Finally, the ideomotor approach (Paulus, 2012) proposes that action mirroring contributes to action understanding via motor-related representations and modulation of attentional processes, without involving the conceptual ascription of goals and mental states to others. These theoretical approaches make fundamentally different assumptions and predictions about how action mirroring might be related to action understanding. The direct-matching hypothesis suggests that motor simulations play a causal role in our ability to ascribe mental states to others. Corollaries are that the development of action understanding follows the development of motor abilities and that hindering motor simulations hampers action understanding. The teleological theory, in contrast, predicts that action mirroring is a consequence of goal understanding, which is already in place early in development. Finally, the ideomotor approach suggests that people process and understand others' behaviors on different levels of complexity. Here, action mirroring contributes to more simple forms of action understanding that are already present in younger children and is conceptually distinct from higher-order levels of understanding (e.g., mental state attribution), which show more prolonged developmental trajectories.

This special issue of the British Journal of Developmental Psychology (BJDP) includes both empirical and theoretical contributions that explore questions pertaining to the development of action mirroring. A particular strength of this body of work comes from the diverse perspectives and methodologies represented, with the aim of understanding action mirroring in the course of development. The contributions to this special issue comprise behavioral 
studies of imitation and visual attention/eye tracking as well as neural investigations (i.e., EEG desynchronization, event-related potentials) of action mirroring.

In the following sections, we briefly introduce the contributions and situate them in the theoretical debate.

\section{Contributions in the current special issue}

Quadrelli and Turati (2016) review and critically analyze different models about the origins and early development of action mirroring, including the debated contribution of mirror mechanisms to action understanding. The authors propose a neuroconstructivist framework as a novel account that yields hypotheses consistent with current findings. According to this framework, mirroring mechanisms emerge from experience-expectant processes and action understanding involves a multilayer structure with an interplay between top-down and bottom-up processes.

Yoo, Cannon, Thorpe, and Fox (2016) investigated the emergence of a neural system that supports the coupling of action perception and execution (i.e., neural mirroring). They found age-related changes in EEG desynchronization during the perception of means-end actions with 9-month-olds exhibiting greater desynchronization than 12-month-olds. Importantly, their findings indicated that emerging grasping skills were associated with desynchronization during action perception at 12 , but not 9 , months.

Boyer and Bertenthal (2016) used an observational A-not-B task to examine the role of prior visual experience (i.e., watching others' ipsilateral/contralateral reaches) on infants' subsequent search performance. Nine-month-olds who were familiarized with contralateral reaching, subsequently searched incorrectly. This pattern was not found for infants familiarized with ipsilateral reaching, presumably because the movement-specific visual experience primed infants' motor representations (i.e., covert imitation).

Gampe, Prinz, and Daum (2016) examined associations between goal prediction and imitation in 12- to 30-month-old children. They found that predictive gaze shifts to an action goal were related to infants' subsequent imitation of the multistep action sequence. Interestingly, this association was only exhibited for one of the two action sequences, indicating task specificity of action mirroring during early childhood.

Meyer, Braukmann, Stapel, Bekkering, and Hunnius (2016) investigated whether and when in development neural mirroring systems relate to the monitoring of others' action errors. Although 9- and 14-month-olds exhibited EEG suppression related to motor activity during action execution and perception, only EEG suppression related to visual activity differentiated others' action errors. In contrast, adult participants exhibited action error sensitivity in EEG motor activity suppression.

Galilee and McCleery (2016) measured event-related potentials (ERPs) to examine the neural mechanisms of self-other tactile perception in 4- to 5-year-olds. Children exhibited differences in ERPs as a function of touch (touch vs. non-touch) and stimulus type (human vs. non-human), similar to previous evidence with adults. The authors consider these 
findings to indicate that young children exhibit tactile mirroring mechanisms, providing evidence that mirroring goes beyond the mirroring of simple actions.

Reddy and Uithol (2016) provide a critical analysis of the role of action mirroring in action understanding, proposing that building action understanding on action mirroring might be problematic. More precisely, they argue that action understanding is a dynamic process that is not captured by action mirroring. The authors review current evidence of action understanding, proposing that action engagement explanations better account for many of these findings.

Likewise, an empirical contribution to the special issue examined potential limitations of the role of action mirroring in action understanding. Choisdealbha, Westermann, Dunn, and Reid (2016) used eye tracking to determine whether it was possible to dissociate associative and motor aspects of infant action understanding. They measured 16-month-olds' looking behavior to images of actors holding dual-function tools in manners congruent or incongruent with their goals. When the motor components (i.e., hand postures) were held constant, infants could use solely associative processes to understand the actor's goals.

In a series of studies, Subiaul, Patterson, and Barr (2016) examined the cognitive structure of imitation (action mirroring; Subiaul, Patterson, Schilder, Renner, \& Barr, 2015) and goal emulation (intention mirroring), trying to demarcate action mirroring from related phenomena and processes. Their findings indicate that for each type of mirroring, cognitive structure varies as a function of both domain and task demands. The authors concluded that developmental changes in emulation were associated with more domain-general processes as compared to developmental changes in imitation.

\section{Acknowledgments}

Manuscript preparation was supported by grant HD081333 to KC from the Eunice Kennedy Shriver National Institute of Child Health and Human Development (NICHD), grant DC013864 from the National Institute of Deafness and Other Communication Disorders (NIDCD; PI J. Irwin), grant PA 2302/6-1 by the German Research Foundation (Deutsche Forschungsgemeinschaft; DFG) to MP, and grant 2376 by the German-Israeli-Foundation (GIF) to MP. The content of this manuscript is solely the responsibility of the authors and does not necessarily represent the official views of the National Institutes of Health, NICHD, NIDCD, DFG, or GIF.

\section{References}

Bonini L, Ferrari PF. Evolution of mirror systems: A simple mechanism for complex cognitive functions. Annals of the New York Academy of Sciences. 2011; 1225:166-175.10.1111/j. 1749-6632.2011.06002.x [PubMed: 21535003]

Boyer TW, Bertenthal BI. Infants' observation of others' actions: Brief movement-specific visual experience primes motor representations. British Journal of Developmental Psychology. 201610.1111/bjdp.12103

Choisdealbha AN, Westermann G, Dunn K, Reid V. Dissociating associative and motor aspects of action understanding: Processing of dual-ended tools by 16-month-old infants. British Journal of Developmental Psychology. 201610.1111/bjdp.12116

Csibra, G. Action mirroring and action understanding: An alternative account. In: Haggard, P.; Rosetti, Y.; Kawate, M., editors. Sensorimotor Foundations of Higher Cognition: Attention and Performance. Vol. 22. Oxford: Oxford University Press; 2007. p. 435-480. 
Csibra G, Gergely G. 'Obsessed with goals': Functions and mechanisms of teleological interpretation of actions in humans. Acta Psychologica. 2007; 124:60-78.10.1016/j.actpsy.2006.09.007 [PubMed: 17081489]

Cuevas K, Cannon EN, Yoo K, Fox NA. The infant EEG mu rhythm: Methodological considerations and best practices. Developmental Review. 2014; 34:26-43.10.1016/j.dr.2013.12.001 [PubMed: 24563573]

Ebisch SJH, Perrucci MG, Ferretti A, Del Gratta C, Romani GL, Gallese V. The sense of touch: embodied simulation in a visuotactile mirroring mechanism for observed animate or inanimate touch. Journal of Cognitive Neuroscience. 2008; 20:1611-1623.10.1162/jocn.2008.20111 [PubMed: 18345991]

Ferrari PF, Tramacere A, Simpson EA, Iriki A. Mirror neurons through the lens of epigenetics. Trends in Cognitive Sciences. 2013; 17:450-457.10.1016/j.tics.2013.07.003 [PubMed: 23953747]

Galilee A, McCleery JP. Neural mechanisms of the observation of human and non-human object touch in children: An event-related potential study. British Journal of Developmental Psychology. 201610.1111/bjdp.12119

Gampe A, Prinz W, Daum MM. Measuring action understanding: Relations between goal prediction and imitation. British Journal of Developmental Psychology. 201610.1111/bjdp.12125

Heyes CM. Where do mirror neurons come from? Neuroscience and Biobehavioral Reviews. 2010; 34:575-583.10.1016/j.neubiorev.2009.11.007 [PubMed: 19914284]

Heyes C. Tinbergen on mirror neurons. Philosophical Transactions of the Royal Society B. 2014; 369:20130180.10.1098/rstb.2013.0180

Jones SS. Imitation in infancy: The development of mimicry. Psychological Science. 2007; 18:593599.10.1111/j.1467-9280.2007.01945.x [PubMed: 17614867]

Jones SS. The development of imitation in infancy. Philosophical Transactions of the Royal Society B. 2009; 364:2325-2335.10.1098/rstb.2009.0045

Lepage J-F, Theoret H. The mirror neuron system: grasping others` actions from birth? Developmental Science. 2007; 10:513-529.10.1111/j.1467-7687.2007.00631.x [PubMed: 17683336]

Meltzoff AN. „Like me" : a foundation for social cognition. Developmental Science. 2007; 10:126134.10.1111/j.1467-7687.2007.00574.x [PubMed: 17181710]

Meyer M, Braukmann R, Stapel JC, Bekkering H, Hunnius S. Monitoring others' errors: The role of the motor system in early childhood and adulthood. British Journal of Developmental Psychology. 201610.1111/bjdp.12101

Over H, Carpenter M. Putting the social into social learning: Explaining both selectivity and fidelity in children's copying behavior. Journal of Comparative Psychology. 2012; 126:182-192.10.1037/ a0024555 [PubMed: 21767011]

Paulus M. Imitation in infancy: Conceptual considerations. Theory \& Psychology. 2011; 21:849855.10.1177/0959354310395990

Paulus M. Action mirroring and action understanding: An ideomotor and attentional account. Psychological Research. 2012; 76:760-767.10.1007/s00426-011-0385-9 [PubMed: 22057651]

Paulus M. How and why do infants imitate? An ideomotor approach to social and imitative learning in infancy (and beyond). Psychonomic Bulletin \& Review. 2014; 21:1139-1156.10.3758/ s13423-014-0598-1 [PubMed: 24578090]

Quadrelli E, Turati C. Origins and development of mirroring mechanisms: A neuroconstructivist framework. British Journal of Developmental Psychology. 201610.1111/bjdp.12110

Reddy V, Uithol S. Engagement: Looking beyond the mirror to understand action understanding. British Journal of Developmental Psychology. 201610.1111/bjdp.12106

Rizzolatti G, Arbib MA. Language within our grasp. Trends in Neurosciences. 1998; 21:188194.10.1016/S0166-2236(98)01260-0 [PubMed: 9610880]

Rizzolatti G, Craighero L. The mirror-neuron system. Annual Review Neurosciences. 2004; 27:169192.10.1146/annurev.neuro.27.070203.144230

Rizzolatti G, Fogassi L, Gallese V. Neurophysiological mechanisms underlying the understanding and imitation of action. Nature Reviews Neuroscience. 2001; 2:661-670.10.1038/35090060 [PubMed: 11533734] 
Subiaul F, Patterson EM, Barr R. The cognitive structure of goal emulation during the preschool years. British Journal of Developmental Psychology. 201610.1111/bjdp.12111

Subiaul F, Patterson EM, Schilder B, Renner E, Barr R. Becoming a high fidelity-super-imitator: What are the contributions of social and individual learning? Developmental Science. 2015; 18:1025-1035.10.1111/desc.12276 [PubMed: 25545051]

Tomasello, M. The cultural origins of human cognition. Cambridge: Harvard University Press; 1999.

Yoo KH, Cannon EN, Thorpe SG, Fox NA. Desynchronization in EEG during perception of meansend actions and relations with infants' grasping skill. British Journal of Developmental Psychology. 201610.1111/bjdp.12115 